\title{
SOLUTION OF THE HADAMARD PROBLEM IN THE CLASS OF STEPWISE GAUGE-EQUIVALENT DEFORMATIONS OF HOMOGENEOUS DIFFERENTIAL OPERATORS WITH CONSTANT COEFFICIENTS
}

\author{
S. P. KHEKALO
}

\begin{abstract}
In the paper, all nontrivial Huygens stepwise gauge-equivalent deformations for a priori Huygens homogeneous differential operators with constant coefficients are described explicitly. A condition is obtained under which an operator in the class of stepwise gauge-equivalent operators is Huygens, and new examples are given of iso-Huygens deformations of radial homogeneous differential operators of higher order.
\end{abstract}

\section{$\S 1$ Introduction. Statement of the Problem}

The problem of describing the differential equations (operators) satisfying the Huygens principle is well known in the literature as Hadamard's problem (see, e.g., [1]-3] and the references therein).

A differential operator satisfies the Huygens principle (in the sense of Hadamard) if it has a fundamental solution with support of positive codimension.

After Hadamard's work, it had long been a common opinion that the Huygens principle is a unique phenomenon inherent only to the wave operator (or to its elementary transformations). However, Stellmacher 4] constructed a nontrivial example of a Huygens operator by adding a potential of a special type to the wave operator. In modern terms, he performed an iso-Huygens deformation of the wave operator by a potential with a second order singularity in the plane.

Later, Stellmacher and Lagnese [5] obtained all possible iso-Huygens deformations of the wave operator by potentials depending on one variable. It turned out that all such potentials (Lagnese-Stellmacher potentials) can be obtained from the zero potential by finitely many Darboux transformations of the one-dimensional Schrödinger operator; see [6, 2, 3].

Actually, from that point on, a new line of investigations in Hadamard's problem started to develop, related to performing nontrivial iso-Huygens deformations of a priori Huygens operators (see, e.g., [3], 7]-[10]).

Presently, the Hadamard problem is well studied for second order operators with arbitrary coefficients (especially in four-dimensional space; see [2, 3]).

For higher order operators, Hadamard's problem is poorly studied (see, e.g., [11, 12]). No attempts to extend the Hadamard construction directly to higher order operators have been a success [13].

2000 Mathematics Subject Classification. Primary 53A04; Secondary 52A40, 52A10.

Key words and phrases. Hadamard problem, Huygens principle, homogeneous operators, deformations, Riesz kernels, gauge equivalence, stepwise gauge equivalence.

Supported by the president of RF (grant no. MK-2195.2007.1) and by RFBR (grant no. 07-01-00085). 
In the papers $[3,7,8,10$, based on the theory of gauge-equivalent operators and intertwining operators with spectral parameters, Berest, Veselov, and Molchanov constructed an analog of the Hadamard Ansatz for deformed operators of arbitrary orders. This construction made it possible to obtain iso-Huygens deformations of the wave operator by the Calogero-Moser potentials [3].

It is well known that not all Huygens equations can be transformed into equations with constant coefficients in the principal parts. Examples of such equations are provided by the Günther equations 14 and the Semenov-Tyan-Shanskiu hyperbolic systems of wave equations with "multidimensional time" [15. Using the results of [15], Helgason [16] described an important relationship between the Huygens principle and problems of integral geometry. A link between the validity of the Huygens principle and the possibility for obtaining local inversion formulas for the Radon transformation was also discussed in [17, and that between the Huygens principle and the validity of the Fuglede formula for the integral transformation along planes was discussed in [18].

We note that the Hadamard criterion for the finiteness of a series-Ansatz [1], the Petrovskiu criterion for the existence of a lacuna [19], and the links established between the Huygens principle and a local inversion formula for the Radon transformation [15, 16] allow one only to determine whether a given operator is Huygens and give no way to construct new Huygens operators.

In the present paper, we use the approach of Stellmacher-Lagnese-Berest-Veselov to solve the Hadamard problem in the class of stepwise gauge-equivalent deformations of homogeneous differential operators with constant coefficients. Thus, we are interested in the situation where the basic space related to an equation (operator) of an arbitrary order is locally conformally flat (in this case, the coefficients of the principal part of the operator can be regarded as constants).

In this paper, the basic methods and notions do not depend on the fact that the coefficients of the principal part of the operator in question are constant. Therefore, there is some hope that the results of the paper can be extended to the case of variable coefficients.

The author is grateful to the participants of V. M. Babich's seminar on mathematical physics and to Semenov-Tyan-Shanskii for an interesting and important discussion of problems touched upon in this paper; to Yu. Yu. Berest and A. P. Veselov, whose work provided a basis for the author's results; and to the reviewer for significant remarks that improved the exposition in $\S \S 4,5$, and 8 .

\section{§2. Gauge-equivalent operators}

Specific examples of classes of differential operators satisfying the so-called gauge equivalence property were presented in the papers [3], 7]- 9], where the verification of this property was based on the method of intertwining operators with spectral parameters and is fairly difficult. The problem of describing more or less general constructions of classes of gauge-equivalent differential operators was posed in the paper [8]. An attempt at constructing a gauge equivalence class for hyperbolic operators was made in [10.

In the present paper, we use the notion of stepwise gauge-equivalence of operators 20], which is a direct analog of the Darboux transformation of the one-dimensional Schrödinger operator [2, 3, 6, and give an explicit construction of deformations of homogeneous differential operators with constant coefficients that are gauge equivalent to a given operator.

On $\mathbb{R}^{N}, N \geq 2$, we consider a homogeneous differential operator $\mathcal{L}_{0}$ of order $K \geq 2$ with constant coefficients. We assume that the operator $\mathcal{L}_{0}$ admits a Riesz kernel. This

means [8] that there exists a family of distributions $\Phi_{0}^{(\lambda)}$ on $\mathbb{R}^{N}$ meromorphic in the 
domain $\operatorname{Re} \lambda>\lambda_{0}, \lambda_{0}<0$ ( $\lambda$ is a complex parameter), and satisfying the conditions

$$
\mathcal{L}_{0} \Phi_{0}^{(\lambda)}=\Phi_{0}^{(\lambda-1)}, \quad \Phi_{0}^{(0)}=\delta
$$

( $\delta$ is the Dirac delta function). A formal method for constructing a Riesz kernel of $\mathcal{L}_{0}$ in the form of Fourier integrals was given in [8]. There are many specific cases where this method yields explicit expressions for Riesz kernels; see [3, 21].

By a deformation $\mathcal{L}$ of an operator $\mathcal{L}_{0}$ we mean an operator

$$
\mathcal{L}=\mathcal{L}_{0}+\cdots
$$

obtained from $\mathcal{L}_{0}$ by adding differential operators of smaller order with variable coefficients (and admitting no reduction to $\mathcal{L}_{0}$ by elementary transformations; see [22, 1, 2]).

Let $\mathcal{L}_{k}, k \in \mathbb{Z}_{+}$, be deformations of $\mathcal{L}_{0}$ depending on a parameter and satisfying the condition

$$
\left.\mathcal{L}_{k}\right|_{k=0} \equiv \mathcal{L}_{0}
$$

We recall [3] that an operator $\mathcal{L}_{k}$ is $M$-gauge equivalent to $\mathcal{L}_{0}$ via a nonzero smooth function $f_{k}$ if

$$
\operatorname{ad}_{\mathcal{L}_{k}, \mathcal{L}_{0}}^{M+1} f_{k}=0, \quad M=M(k) \in \mathbb{N},
$$

where the skew adjoint action operator $\operatorname{ad}_{A, B}^{M} C$ is defined by the recurrence relation

$$
\operatorname{ad}_{A, B}^{M} C=\operatorname{ad}_{A, B}^{M-1}(A C-C B), \quad \operatorname{ad}_{A, B}^{0} C=C .
$$

Theorem 1 (the Berest-Veselov-Molchanov Ansatz [8]). Suppose the operator $\mathcal{L}_{k}$ is $M$-gauge equivalent to $\mathcal{L}_{0}$ by a function $f_{k}$. Then the family of distributions

$$
\left\{\left.\mathcal{H}_{\partial_{t}}^{M}\left(f_{k}\right) \Phi_{k}^{(\lambda)}(x, \xi ; t)\right|_{t=0}, x, \xi \in \mathbb{R}^{N} ; t \in \mathbb{R}\right\}_{\lambda \in \mathbb{C}},
$$

where

$$
\mathcal{H}_{\partial_{t}}^{M}\left(f_{k}\right)=\sum_{s=0}^{M} \frac{(-1)^{s}}{s !}\left(\operatorname{ad}_{\mathcal{L}_{k}, \mathcal{L}_{0}}^{s} f_{k}\right) \frac{\partial^{s}}{\partial t^{s}}
$$

and

$$
\Phi_{k}^{(\lambda)}(x, \xi ; t)=\frac{1}{f_{k}(\xi)} \sum_{i=0}^{Q}\left(\begin{array}{c}
\lambda+i-1 \\
i
\end{array}\right) \Phi_{0}^{(\lambda+i)}(x-\xi) t^{i}
$$

( $Q$ is sufficiently large) gives a Riesz kernel for $\mathcal{L}_{k}$ :

$$
\begin{aligned}
\mathcal{L}_{k}\left(\left.\mathcal{H}_{\partial_{t}}^{M}\left(f_{k}\right) \Phi_{k}^{(\lambda)}(x, \xi ; t)\right|_{t=0}\right) & =\left.\mathcal{H}_{\partial_{t}}^{M}\left(f_{k}\right) \Phi_{k}^{(\lambda-1)}(x, \xi ; t)\right|_{t=0}, \\
\left.\mathcal{H}_{\partial_{t}}^{M}\left(f_{k}\right) \Phi_{k}^{(0)}(x, \xi ; t)\right|_{t=0} & =\delta(x-\xi) .
\end{aligned}
$$

As was noted above, it is difficult to verify condition (1) for specific deformations $\mathcal{L}_{k}$. It is also unclear why the deformations $\mathcal{L}_{k}$ must have a specific form and whether gauge-equivalent deformations can be constructed for every homogeneous operator $\mathcal{L}_{0}$.

In this connection, the following problem arises: construct an algorithm for finding the coefficients of the deformation $\mathcal{L}_{k}$ having a Riesz kernel (2).

A special case of this problem was solved in the paper [20] where, on the basis of Fourier's method of separation of variables and the conditions of stepwise gauge equivalence for operators, deformations of the operator $\mathcal{L}_{0}$ in a given direction were constructed, with coefficients of the deformations depending on one variable.

In the present paper, the above problem is solved with the help of a necessary and sufficient condition for stepwise gauge equivalence of operators. Moreover, our criterion 
leads to a specific form of these deformations (up to shifts in the variable). Preparatory work was done in 23], where the problem on stepwise gauge equivalence of deformations $\mathcal{L}_{k}, k \in\{0\} \cup \mathbb{N}$, was reduced to the problem of solving an overdetermined system of partial differential equations for the coefficients of the deformations and for the function $f_{k}$ (see equation (1)).

\section{§3. Deformations. Riesz kernels}

A homogeneous differential operator $\mathcal{L}_{0}$ of order $K$ with constant coefficients $a_{\alpha_{1}, \ldots, \alpha_{K}}$, $\alpha_{i}=1, \ldots, N, i=1, \ldots, K$, is a homogeneous $K$-form in the partial derivatives $\partial_{\alpha_{i}}=$ $\partial / \partial x_{\alpha_{i}}$

$$
\mathcal{L}_{0}=\sum_{\alpha} a_{\alpha_{1}, \ldots, \alpha_{K}} \partial_{\alpha_{1}} \cdots \partial_{\alpha_{K}} 1
$$

We introduce the following notation: $($.$) is a binomial coefficient; \langle\cdot, \cdot\rangle$ is the standard scalar product in $\mathbb{R}^{N} ;(k, s)=k(k+1) \cdot \ldots \cdot(k+s-1)$ is the Pochhammer symbol $(k, s \in\{0\} \cup \mathbb{N},(k, 0) \equiv 1)$.

We choose an arbitrary nonzero vector parameter

$$
A=\left(A_{1}, \ldots, A_{N}\right) \in \mathbb{R}^{N}
$$

and, for $s=1, \ldots, K$, consider the sums

$$
q_{s}(\alpha, A)=\sum_{\alpha_{K-s+1}, \ldots, \alpha_{K}} a_{\alpha_{1}, \ldots, \alpha_{K}} A_{\alpha_{K-s+1}} \cdots A_{\alpha_{K}} .
$$

For $s=0$, we put

$$
q_{0} \equiv q_{0}(\alpha, A)=a_{\alpha_{1}, \ldots, \alpha_{K}} .
$$

Obviously, the operators

$$
\mathcal{R}_{k}=\sum_{s=0}^{K} \frac{(-1)^{s-1}(s-1)\left(\begin{array}{l}
K \\
s
\end{array}\right)(k, s)}{\langle A, x\rangle^{s}} \sum_{\alpha} q_{s}(\alpha, A) \partial_{\alpha_{1}} \cdots \partial_{\alpha_{K-s}}
$$

provide a sequence of deformations of the operator $\mathcal{L}_{0}$. In other words, $\mathcal{R}_{0}=\mathcal{L}_{0}$ and

$$
\mathcal{R}_{k}=\mathcal{L}_{0}+\text { smaller order terms. }
$$

Proposition 1. Let $\Phi_{0}^{(\lambda)}$ be a Riesz kernel of $\mathcal{L}_{0}$. Then, for each $k \in \mathbb{Z}_{+}$, the family of distributions

$$
\left\{\left.\mathcal{H}_{\partial_{t}}^{k}\left(\langle A, x\rangle^{k}\right) \Phi_{k}^{(\lambda)}(x, \xi ; t)\right|_{t=0}, \quad x, \xi \in \mathbb{R}^{N} ; t \in \mathbb{R}\right\}_{\lambda \in \mathbb{C}}
$$

where

$$
\mathcal{H}_{\partial_{t}}^{k}\left(\langle A, x\rangle^{k}\right)=\sum_{s=0}^{k} \frac{(-1)^{s}}{s !}\left(\operatorname{ad}_{\mathcal{R}_{k}, \mathcal{L}_{0}}^{s}\langle A, x\rangle^{k}\right) \frac{\partial^{s}}{\partial t^{s}}
$$

and

$$
\Phi_{k}^{(\lambda)}(x, \xi ; t)=\frac{1}{\langle A, \xi\rangle^{k}} \sum_{i=0}^{\infty}\left(\begin{array}{c}
\lambda+i-1 \\
i
\end{array}\right) \Phi_{0}^{(\lambda+i)}(x-\xi) t^{i},
$$

is a Riesz kernel of $\mathcal{R}_{k}$.

\footnotetext{
${ }^{1}$ We deliberately use this kind of notation for an operator (in contrast to the multi-index notation). This will allow us to introduce some constructions and simplify calculations in the proof of the main theorem.
} 
The proof is based on Theorem 1 in [8], mentioned in the Introduction, and on the condition for the operators $\mathcal{R}_{k}$ and $\mathcal{L}_{0}$ to be $k$-gauge equivalent via the function $\langle A, x\rangle^{k}$ :

$$
\operatorname{ad}_{\mathcal{R}_{k}, \mathcal{L}_{0}}^{k+1}\langle A, x\rangle^{k}=0 .
$$

The proof of the latter relation is based on the property of stepwise gauge equivalence for the operators $\mathcal{R}_{k}, k \in \mathbb{Z}_{+}[23]$.

\section{§4. Stepwise GaUge EQUivalence}

As above, let $\mathcal{L}_{k}, k \in \mathbb{Z}_{+}$, be deformations of the operator $\mathcal{L}_{0}$, and let $s$ be a positive integer.

Definition 1. The operators $\mathcal{L}_{k}, k=0,1, \ldots, s$, satisfy the condition of stepwise gauge equivalence via one and the same function $f$ if, for all $k=1, \ldots, s$, the following relation is valid:

$$
\operatorname{ad}_{\mathcal{L}_{k}, \mathcal{L}_{k-1}}^{1+1} f=0
$$

Thus, by definition, two operators with neighboring values of the parameter are 1gauge equivalent via $f$. (Actually, this definition was introduced in Theorem 1 in [23].)

Proposition 2 [23]. If the operators $\mathcal{L}_{k}, k=0,1, \ldots, s$, satisfy the condition of stepwise gauge equivalence via a function $f$, then $\mathcal{L}_{s}$ is s-gauge equivalent to $\mathcal{L}_{0}$ via the function $f^{s}$ :

$$
\operatorname{ad}_{\mathcal{L}_{k}, \mathcal{L}_{k-1}}^{1+1} f=0, \quad k=1, \ldots, s \Longrightarrow \operatorname{ad}_{\mathcal{L}_{s}, \mathcal{L}_{0}}^{s+1} f^{s}=0
$$

Thus, to prove that conditions (4) are satisfied, it suffices to verify conditions (5) for the stepwise gauge equivalence of the operators (3). In the following theorem, we present necessary and sufficient conditions under which deformations of homogeneous differential operators with constant coefficients are gauge equivalent.

Theorem 2. The operators $\mathcal{L}_{k}$ satisfy the condition of stepwise gauge equivalence if and only if, up to translations of the variable $x \in \mathbb{R}^{N}$, they are of the form (3):

$$
\mathcal{L}_{k} \equiv \mathcal{R}_{k}=\sum_{j=0}^{K} \frac{(-1)^{j-1}(j-1)\left(\begin{array}{c}
K \\
j
\end{array}\right)(k, j)}{\langle A, x\rangle^{j}} \sum_{\alpha} q_{j}(\alpha, A) \partial_{\alpha_{1}} \cdots \partial_{\alpha_{K-j}},
$$

where $K$ is the degree of the operator $\mathcal{L}_{0}$.

Proof. Let

$$
\mathcal{L}_{0}=\sum_{\alpha,|\alpha|=K} a_{\alpha} \partial^{\alpha}
$$

where now $\alpha=\left(\alpha_{1}, \ldots, \alpha_{N}\right)$ is a multi-index of nonnegative integers, $|\alpha|=\alpha_{1}+\cdots+\alpha_{N}$, $\partial=\partial^{|\alpha|} / \partial x_{1}^{\alpha_{1}} \cdots \partial x_{N}^{\alpha_{N}}$.

By the necessary condition for gauge equivalence of operators, the principal symbols of these operators must coincide [23]. Therefore, we define a deformation of $\mathcal{L}_{0}$, depending on the parameter $k \in \mathbb{Z}_{+}$, by the equation

$$
\mathcal{L}_{k}=\sum_{\alpha,|\alpha| \leq K} u_{\alpha}^{k}(x) \partial^{\alpha}
$$

where the coefficients $u_{\alpha}^{k}(x)$ are smooth almost everywhere and satisfy the conditions

$$
u_{\alpha}^{0}(x)=\left\{\begin{array}{ll}
a_{\alpha}, & |\alpha|=K, \\
0, & |\alpha|<K ;
\end{array} \quad u_{\alpha}^{k}(x)=u_{\alpha}^{0}(x)=a_{\alpha}, \quad|\alpha|=K .\right.
$$


Next, for every smooth function $f \not \equiv 0$, we put

$$
\begin{gathered}
g_{\alpha}^{k}(f) \equiv g_{\alpha}^{k}=\sum_{\beta \geq \alpha,|\beta| \leq K}\left(\begin{array}{c}
\beta \\
\alpha
\end{array}\right) u_{\beta}^{k}\left(\partial^{\beta-\alpha} f\right)-u_{\alpha}^{k-1} f, \quad|\alpha| \leq K, \\
r_{\alpha \beta}^{k}(f) \equiv r_{\alpha \beta}^{k}=\sum_{\gamma \geq \alpha,|\gamma| \leq K}\left(\begin{array}{c}
\gamma \\
\alpha
\end{array}\right)\left[u_{\gamma}^{k}\left(\partial^{\gamma-\alpha} g_{\beta}^{k}\right)-g_{\gamma}^{k}\left(\partial^{\gamma-\alpha} u_{\beta}^{k-1}\right)\right], \quad|\alpha|,|\beta| \leq K,
\end{gathered}
$$

where the binomial coefficient is defined on multi-indices as the product of binomial coefficients on the corresponding subindices, and the partial ordering of multi-indices is defined by the rule $\beta \geq \alpha \Leftrightarrow \beta_{i} \geq \alpha_{i}$ for all $i=1, \ldots, N$.

In [23], it was proved that condition (5) of stepwise gauge equivalence can be rewritten as the overdetermined system

$$
\left\{\begin{array}{cl}
\sum_{\alpha \geq \gamma,|\alpha| \leq K} r_{\alpha, \gamma-\alpha}^{k}=0, & |\gamma| \leq K, \\
\sum_{\alpha \geq \gamma,|\gamma|-K \leq|\alpha| \leq K} r_{\alpha, \gamma-\alpha}^{k}=0, & 1+K \leq|\gamma| \leq 2 K,
\end{array}\right.
$$

consisting of $\left(\begin{array}{c}2 K+N \\ N\end{array}\right)$ nonlinear partial differential equations, for $u_{\alpha}^{k}(x)$ and $f$. As was proved in [23, the function $f$ must be a linear form (up to affine shifts),

$$
f(x) \equiv f_{A}(x)=\langle A, x\rangle, \quad A, x \in \mathbb{R}^{N} .
$$

We represent the deformation (6) in the following form similar to (3):

$$
\mathcal{R}_{k}=\sum_{j=0}^{K} \sum_{\alpha} u_{j}^{k}(\alpha, x) \partial_{\alpha_{1}} \cdots \partial_{\alpha_{K-j}}
$$

Solving system (7) for such operators, with $f(x)=\langle A, x\rangle$, we see that the coefficients $u_{j}^{k}(\alpha, x)$ must have the form

$$
u_{j}^{k}(\alpha, x)=\frac{c_{j}^{k}\left(\alpha ; q_{0} ; A\right)}{\langle A, x\rangle^{j}}, \quad c_{0}^{k}\left(\alpha ; q_{0} ; A\right)=q_{0} .
$$

Thus, system (7) can be transformed into an overdetermined system of nonlinear equations for the constants $c_{j}^{k}\left(\alpha ; q_{0} ; A\right)$.

Next, the calculation of the commutator

$$
\left[\partial_{\alpha_{1}} \cdots \partial_{\alpha_{K-j}},\langle A, x\rangle^{l}\right], \quad l \in \mathbb{Z},
$$

shows that the $c_{j}^{k}\left(\alpha ; q_{0} ; A\right)$ look like this:

$$
c_{j}^{k}\left(\alpha ; q_{0} ; A\right)=p_{j}(k, j) q_{j}(\alpha ; A),
$$

where the $p_{j}=p(j)$ are constants depending only on $j$, and as before, $(k, j)$ is the Pochhammer symbol.

Consequently, the operators $\mathcal{L}_{k}$ satisfy (up to translations) the condition of stepwise gauge equivalence if and only if

$$
\mathcal{L}_{k}=\sum_{j=0}^{K} \frac{p_{j}(k, j)}{\langle A, x\rangle^{j}} \sum_{\alpha} q_{j}(\alpha, A) \partial_{\alpha_{1}} \cdots \partial_{\alpha_{K-j}} .
$$


We introduce the notation

$$
\begin{aligned}
r_{j} & =(K-j) p_{j}+(j+1) p_{j+1}, \\
P_{j l}(k ; K) & =\sum_{r=0}^{j}(-1)^{r} p_{j-r}(k, j-r)(l, r)\left(\begin{array}{c}
K-j+r \\
r
\end{array}\right), \\
Q_{j l}(k ; K) & =\sum_{r=0}^{l}(-1)^{r} r_{l-r}(k, l-r)(j, r)\left(\begin{array}{c}
K-l+r-1 \\
r
\end{array}\right)
\end{aligned}
$$

and find the constants $p_{j}$.

Let $f(x)=\langle A, x\rangle$, as before. A direct calculation (it is faster to use a computer algebra package) shows that, first,

$$
\operatorname{ad}_{\mathcal{L}_{k}, \mathcal{L}_{k-1}} f=\sum_{j=0}^{K-1} \frac{(k, j) r_{j}}{\langle A, x\rangle^{j}} \sum_{\alpha} q_{j+1}(\alpha ; A) \partial_{\alpha_{1}} \cdots \partial_{\alpha_{K-j-1}},
$$

and second,

$$
\begin{aligned}
\operatorname{ad}_{\mathcal{L}_{k}, \mathcal{L}_{k-1}}^{2} f \equiv & \mathcal{L}_{k}\left(\operatorname{ad}_{\mathcal{L}_{k}, \mathcal{L}_{k-1}} f\right)-\left(\operatorname{ad}_{\mathcal{L}_{k}, \mathcal{L}_{k-1}} f\right) \mathcal{L}_{k-1} \\
= & \sum_{j=0}^{K} \sum_{l=0}^{K-1} \frac{r_{l}(k, l) P_{j l}(k ; K)-p_{j}(k-1, j) Q_{j l}(k ; K)}{\langle A, x\rangle^{l+j}} \\
& \quad \times \sum_{\alpha} \sum_{\beta} q_{j}(\alpha ; A) q_{l+1}(\beta ; A) \partial_{\alpha_{1}} \cdots \partial_{\alpha_{K-j}} \partial_{\beta_{1}} \cdots \partial_{\beta_{K-l-1}} .
\end{aligned}
$$

Thus, the system of equations for the constants $p_{j}$ has the form

$$
\left\{\begin{aligned}
r_{l} \cdot(k, l) \cdot P_{0 l}(k ; K) & -p_{0} \cdot(k-1,0) \cdot Q_{0 l}(k ; K)=0, \\
r_{l} \cdot(k, l) \cdot P_{l+1, l}(k ; K) & -p_{l+1} \cdot(k-1, l+1) \cdot Q_{l+1, l}(k ; K)=0, \\
r_{l} \cdot(k, l) \cdot P_{j l}(k ; K) & -p_{j} \cdot(k-1, j) \cdot Q_{j l}(k ; K) \\
& +r_{j-1} \cdot(k, j-1) \cdot P_{l+1, j-1}(k ; K) \\
& -p_{l+1} \cdot(k-1, l+1) \cdot Q_{l+1, j-1}(k ; K)=0,
\end{aligned}\right.
$$

where $r_{j}=(K-j) p_{j}+(j+1) p_{j+1}, k \in \mathbb{N}, l=0,1, \ldots, K-1, j=1, \ldots, K$.

Since $P_{0 l}(k ; K)=p_{0}$ and $Q_{0 l}(k ; K)=r_{l}(k, l)$, we see that in the first group the equations are identities:

$$
r_{l}(k, l) p_{0}-p_{0} r_{l}(k, l) \equiv 0 .
$$

We consider the second group of equations in (8) for $k=1$. Since

$$
P_{l+1, l}(k ; K)=\sum_{r=0}^{l+1}(-1)^{r} p_{l+1-r}(k, l+1-r)(l, r)\left(\begin{array}{c}
K-l-1+r \\
r
\end{array}\right),
$$

the equations in this group take the form

$$
\sum_{r=0}^{l+1}(-1)^{r} p_{l+1-r}(l+1-r) !(l, r)\left(\begin{array}{c}
K-l-1+r \\
r
\end{array}\right)=0, \quad l=0,1, \ldots, K-1 .
$$

This is a nondegenerate triangular system, and therefore, it has a nontrivial solution unique up to a constant $p_{0} \neq 0$ :

$$
p_{r}=(-1)^{r-1}(r-1)\left(\begin{array}{c}
K \\
r
\end{array}\right) p_{0} .
$$

It is easy to check that (9) satisfies all equations in (8) for arbitrary $k \in \mathbb{N}$. 
It remains to take into account that $q_{0}=a_{\alpha_{1}, \ldots, \alpha_{K}}$ was defined up to multiplication by a constant. Therefore, we can put $p_{0}=1$. Thus, the numbers of the form (9) and only these numbers (up to a common constant multiple) satisfy system (8), and

$$
\mathcal{R}_{k} \equiv \mathcal{L}_{k}=\sum_{j=0}^{K} \frac{(-1)^{j-1}(j-1)\left(\begin{array}{c}
K \\
j
\end{array}\right)(k, j)}{\langle A, x\rangle^{j}} \sum_{\alpha} q_{j}(\alpha, A) \partial_{\alpha_{1}} \cdots \partial_{\alpha_{K-j}} .
$$

The theorem is proved.

\section{$\S 5$. HAdAmard'S PROBLEM}

We apply Theorem 2 to solve Hadamard's problem in the class of stepwise gaugeequivalent deformations of a priori Huygens homogeneous operators with constant coefficients.

Definition 2. A differential operator satisfies the Huygens principle (the strong Huygens principle) if it has a fundamental solution with support of codimension equal to (greater than) 1.

Definition 3. Suppose a differential operator $\mathcal{L}$ satisfies the Huygens principle. The maximal degree $H$ (finite or infinite) for which the operator $\mathcal{L}^{H}$ remains Huygens is called the Huygens index of $\mathcal{L}$.

Example. Consider the wave operator

$$
\square=\frac{\partial^{2}}{\partial t^{2}}-\frac{\partial^{2}}{\partial x_{1}^{2}}-\cdots-\frac{\partial^{2}}{\partial x_{N}^{2}}
$$

on $\mathbb{R}^{1+N}$. For $N$ odd, the wave operator satisfies the Huygens principle, and the operator $\square^{s}$ satisfies the Huygens principle if and only if the odd number $N$ satisfies the inequality $s \leq(N-1) / 2$ (see, e.g., 2, 3]). Thus, the Huygens index of the wave operator is equal to $H=(N-1) / 2$.

The main consequence of Theorems 2 and 1 is the following statement.

Corollary. Let $\mathcal{L}_{0}$ be a homogeneous differential operator of order $K$ with constant coefficients and with Huygens index $H$. The operator $\mathcal{L}_{k}$ is an iso-Huygens stepwise gauge-equivalent deformation of $\mathcal{L}_{0}$ if and only if $k \leq H-1$ and, up to shifts in the variable, we have

$$
\mathcal{L}_{k}=\sum_{j=0}^{K} \frac{(-1)^{j-1}(j-1)\left(\begin{array}{c}
K \\
j
\end{array}\right)(k, j)}{\langle A, x\rangle^{j}} \sum_{\alpha} q_{j}(\alpha, A) \partial_{\alpha_{1}} \cdots \partial_{\alpha_{K-j}}
$$

for some $A \neq 0$.

Proof. The "only if" part. Let $\mathcal{L}_{k}$ be a stepwise gauge-equivalent deformation of $\mathcal{L}_{0}$. By Theorem 2, up to shifts in the variable, $\mathcal{L}_{k}$ has the form indicated in the corollary. By Theorem 1 and Proposition 1, the fundamental solution of $\mathcal{L}_{k}$ at $\xi \in \mathbb{R}^{N},\langle A, \xi\rangle \neq 0$, is given by the Berest-Veselov-Molchanov Ansatz

$$
\mathcal{E}_{k}=\frac{1}{\langle A, \xi\rangle^{k}} \sum_{j=0}^{k}(-1)^{j}\left(\operatorname{ad}_{\mathcal{L}_{k}, \mathcal{L}_{0}}^{j}\langle A, x\rangle^{k}\right) \Phi_{0}^{(1+j)}(x-\xi), \quad\langle A, \xi\rangle \neq 0 .
$$

Here $\Phi_{0}^{(j)}, j=0,1, \ldots, l(l$ is sufficiently large $)$, is defined by the equation

$$
\Phi_{0}^{(j)}=\mathcal{L}_{0}^{l-j} \mathcal{E}_{0}^{l}, \quad \mathcal{L}_{0}^{l} \mathcal{E}_{0}^{l}=\delta .
$$


Next, since $\mathcal{L}_{0}^{H}$ satisfies the Huygens principle, the codimension of the support of the fundamental solution $\mathcal{E}_{k}$ is at least 1 if $1+j \leq H$ for all $j=0, \ldots, k$. It remains to note that differentiation cannot make the dimension of the support larger.

The "if' part. If, up to shifts in the variable, the operator $\mathcal{L}_{k}$ has the form indicated in the corollary, then, first, $\mathcal{L}_{k}$ is a deformation of $\mathcal{L}_{0}$; second, by Theorem $2, \mathcal{L}_{k}$ satisfies the conditions of stepwise gauge equivalence of operators; and third, its fundamental solution $\mathcal{E}_{k}$ has codimension at least 1 if $k \leq H-1$. Consequently, $\mathcal{L}_{k}$ is an iso-Huygens stepwise gauge-equivalent deformation of $\mathcal{L}_{0}$. The proof is complete.

Remark. Every power $r$ of $\mathcal{L}_{k}$ also satisfies the Huygens principle provided $k \leq H-r$. In this case, the Huygens index $H_{k}$ of the deformed operator $\mathcal{L}_{k}$ is related to the Huygens index $H$ of $\mathcal{L}_{0}$ by the formula

$$
H_{k}=H-k
$$

\section{§6. CAYley-GÅRding-Gindikin operators AND THEIR ISO-HUYGENS DEFORMATIONS}

In this section, we give an explicit example of iso-Huygens deformations of the homogeneous hyperbolic Cayley-Gårding-Gindikin operator [24, 12, 9] on the space $S M_{m}$ of symmetric real $(m \times m)$-matrices.

On $S M_{m}$, we consider the hyperbolic Cayley-Gårding-Gindikin operator

$$
\mathcal{L}_{0} \equiv \mathcal{D}_{m}=\operatorname{det}\left(\varepsilon_{i j} \frac{\partial}{\partial a_{i j}}\right), \quad \varepsilon_{i j}= \begin{cases}1, & i=j, \\ \frac{1}{2}, & i \neq j .\end{cases}
$$

The operator $\mathcal{D}_{m}^{p}$ satisfies the Huygens principle if $3 \leq 2 p+1 \leq m$; see [9, 24].

We denote by $V_{m}^{r}$ the submanifold of nonnegative definite matrices of rank $r=$ $0,1, \ldots, m-1$ in $S M_{m}$. We define almost global coordinates on $V_{m}^{r}$. For all matrices $a \in V_{m}^{r}$ except those belonging to a submanifold of smaller codimension, the first $r$ columns are linearly independent and the matrices can be represented in the form

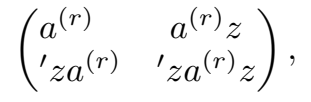

where $a^{(r)}$ is a submatrix of order $r$ and $z$ is an $(r \times(m-r))$-rectangular dependence matrix. On this subset $W$, we introduce the measure

$$
d_{r} a=\frac{1}{\sqrt{\pi}^{r(m-r)} \Gamma_{r}\left(\frac{r}{2}\right)}\left(\operatorname{det} a^{(r)}\right)^{\frac{m-r-1}{2}} d a^{(r)} d z,
$$

which satisfies the quasi-invariance condition

$$
d_{r}\left({ }^{\prime} g a g\right)=|\operatorname{det} g|^{r} d_{r} a, \quad g \in G L(m) .
$$

Definition $4\left([9)\right.$. By the distribution $\delta\left(V_{m}^{r}\right)$ related to the surface $V_{m}^{r}$ in $S M_{m}$, we mean the integral

$$
\left(\delta\left(V_{m}^{r}\right), \varphi\right)=\int_{V_{m}^{r}} \varphi(a) d_{r} a \equiv \int_{W} \varphi(a) d_{r} a,
$$

where $\varphi$ is a Schwartz class function on the space of symmetric real matrices.

Let $A \in S M_{m}$ be a nonzero matrix of parameters, and let

$$
f_{A}(a)=\operatorname{tr}(A a)
$$

be the trace of $A a$. We denote by $\partial^{a}=\left(\varepsilon_{i j} \frac{\partial}{\partial a_{i j}}\right)$ the matrix of partial derivative operators, and by $\partial_{i_{1}, \ldots, i_{s}}^{A}, s=0,1, \ldots, m, 1 \leq i_{1}<\cdots<i_{s} \leq m$, the matrix obtained from $\partial^{a}$ by replacing the rows with indices $i_{1}, \ldots, i_{s}$ by the corresponding rows of $A$. 
If $C G G_{s}\left(A, \partial^{a}\right)$ denotes the homogeneous operator

$$
\sum_{1 \leq i_{1}<\cdots<i_{s} \leq m} \operatorname{det}\left(\partial_{i_{1}, \ldots, i_{s}}^{A}\right)
$$

of order $m-s$, then $C G G_{0}=\mathcal{D}_{m}$ and $C G G_{m}=\operatorname{det}(A)$. We consider the deformation

$$
\mathcal{L}_{k}=\mathcal{D}_{m}+\sum_{s=2}^{m} \frac{(-1)^{s-1}(s-1)(k, s)}{f_{A}^{s}(a)} C G G_{s}\left(A, \partial^{a}\right)
$$

of the Cayley-Gårding-Gindikin operator $\mathcal{D}_{m}=\operatorname{det}\left(\partial^{a}\right)$ with singularities on the planes $\operatorname{tr}(A a)=0$.

Proposition 3. If the parameter $k \in \mathbb{N}$ satisfies the condition

$$
k \leq m-1-2 s, \quad s \in \mathbb{N},
$$

then the strong Huygens principle is fulfilled for the operator $\mathcal{L}_{k}^{s}$. In this case, its principal fundamental solution has the form

$$
\mathcal{E}_{\mathcal{L}_{k}^{s}}=\frac{1}{f_{A}^{k}(\xi)} \sum_{j=0}^{k}(-1)^{j}\left(\begin{array}{c}
s+j-1 \\
j
\end{array}\right)\left(\operatorname{ad}_{\mathcal{L}_{k}, \mathcal{D}_{m}}^{j} f_{A}^{k}(a)\right) \delta\left(V_{m}^{s+j}\right) .
$$

This proposition follows directly from Theorems 1 and 2 .

\section{§7. Iso-Huygens deformations of Cayley-Laplace operators}

In this section, we present a construction of iso-Huygens deformations of a homogeneous nonhyperbolic Cayley-Laplace operator [21, 25] on the space $M_{n, m}$ of rectangular real $(n \times m)$-matrices $(n \geq m)$.

Let $M_{n, m}^{r}$ be the subspace of $M_{n, m}$ consisting of matrices of rank $r=0, \ldots, m-1$. For all matrices $x \in M_{n, m}^{r}$ except those belonging to a subspace of smaller dimension, the first $r$ columns are linearly independent. Let

$$
U=\left\{x=\left(x^{(r)}, x^{(r)} z\right) \mid x^{(r)} \in M_{n, r}, z \in M_{r, m-r}\right\},
$$

where $z$ is the matrix of linear dependence. The group of transformations $x \mapsto \rho x g$, $\rho \in O(n), g \in G L(m)$, acts on $U$. With respect to this action, there is a unique (up to a constant factor) measure on $U$,

$$
d_{r} x=\frac{c_{r} d x^{(r)} d \mu}{\left(\operatorname{det}^{\prime} x^{(r)} x^{(r)}\right)^{(n-m) / 2}}, \quad c_{r}=\pi^{r(r-m-n) / 2} \frac{\Gamma_{r}\left(\frac{n}{2}\right)}{\Gamma_{r}\left(\frac{r}{2}\right)},
$$

that satisfies the following relative invariance condition:

$$
d_{r}(\rho x g)=|\operatorname{det} g|^{r} d_{r} x .
$$

Definition 5. By the delta function $\delta\left(M_{n, m}^{r}\right), r=1, \ldots, m-1$, related to the surface $M_{n, m}^{r}$ in $M_{n, m}$, we mean the distribution

$$
\left(\delta\left(M_{n, m}^{r}\right), \varphi\right)=\int_{M_{n, m}^{r}} \varphi(x) d_{r} x \equiv \int_{U} \varphi(x) d_{r} x,
$$

where $\varphi$ is a Schwartz class function on $M_{n, m}$.

Let $x \in M_{n, m}$ be a rectangular matrix of variables, let $X \in M_{n, m}$ be a nonzero matrix of parameters, and let

$$
f_{X}(x)=\operatorname{tr}\left({ }^{\prime} X x\right)
$$


be the trace of ' $X x$. We denote by $\partial^{x}=\left(\frac{\partial}{\partial x_{i j}}\right)$ the matrix of partial derivative operators, and by $\partial_{i_{1}, \ldots, i_{p}}^{X}, p=0,1, \ldots, m, 1 \leq i_{1}<\cdots<i_{s} \leq m$, the matrix obtained from $\partial^{x}$ by replacing the columns with indices $i_{1}, \ldots, i_{p}$ by the corresponding columns of $X$.

On $M_{n, m}$, we consider the nonhomogeneous nonhyperbolic Cayley-Laplace operator $[9]$

$$
\mathcal{L}_{0} \equiv \Delta_{m}=\operatorname{det}\left({ }^{\prime} \partial^{x} \partial^{x}\right) .
$$

If $2 s \leq \min \{m-1, n-m\}$, then the operator $\Delta_{m}^{s}$ satisfies the strong Huygens principle; see $[25]$.

If $C L_{s}\left(X, \partial^{x}\right)$ denotes the homogeneous operator

$$
\sum_{\substack{1 \leq i_{1}<\cdots<i_{p} \leq m \\ 1 \leq j_{1}<\cdots<j_{q} \leq m \\ p+q=s}} \operatorname{det}\left({ }^{\prime} \partial_{i_{1}, \ldots, i_{p}}^{X} \partial_{j_{1}, \ldots, j_{q}}^{X}\right), \quad s=0,1, \ldots, 2 m,
$$

of order $2 m-s$, then $C L_{0}=\Delta_{m}$ and $C L_{2 m}=\operatorname{det}\left({ }^{\prime} X X\right)$. We consider the deformation

$$
\mathcal{L}_{k}=\Delta_{m}+\sum_{s=2}^{2 m} \frac{(-1)^{s-1}(s-1)(k, s)}{f_{X}^{s}(x)} C L_{s}\left(X, \partial^{x}\right)
$$

of the Cayley-Laplace operator $\Delta_{m}=\operatorname{det}\left({ }^{\prime} \partial^{x} \partial^{x}\right)$ with singularities on the planes $\operatorname{tr}\left({ }^{\prime} X x\right)$ $=0$.

Proposition 4. If the parameter $k \in \mathbb{N}$ satisfies the condition

$$
k \leq \frac{1}{2} \min \{m-1, m-n\}-s, \quad s \in \mathbb{N},
$$

then the strong Huygens principle is fulfilled for $\mathcal{L}_{k}^{s}$, and its principal fundamental solution has the form

$$
\begin{aligned}
\mathcal{E}_{\mathcal{L}_{k}^{s}}=\frac{(-1)^{m p}}{\Gamma_{m}\left(\frac{n}{2}\right) f_{X}^{k}(\xi)} & \sum_{j=0}^{k} \frac{(-1)^{(m+1) j} \Gamma_{m}\left(\frac{n-2 s-2 j}{2}\right)}{4^{(s+j) m}} \\
& \times\left(\begin{array}{c}
s+j-1 \\
j
\end{array}\right)\left(\operatorname{ad}_{\mathcal{L}_{k}, \Delta_{m}}^{j} f_{X}^{k}(x)\right) \delta\left(M_{n, m}^{2 s+2 j}\right) .
\end{aligned}
$$

This proposition follows directly from Theorems 1 and 2 .

\section{$\S 8$. The General PRoblem}

As before, let

$$
\mathcal{L}_{0}=\sum_{\alpha} a_{\alpha_{1}, \ldots, \alpha_{K}} \partial_{\alpha_{1}} \cdots \partial_{\alpha_{K}}, \quad \partial_{\alpha_{i}}=\frac{\partial}{\partial x_{\alpha_{i}}}, \quad \alpha_{i}=1, \ldots, N,
$$

be a homogeneous differential operator on $\mathbb{R}^{N}$ of order $K$ with constant coefficients.

We denote by $\mathcal{A}=\left\{A=\left(A_{1}, \ldots, A_{N}\right) \in \mathbb{R}^{N}\right\}$ a system of nonzero vectors in $\mathbb{R}^{N}$ and by $k_{A}: \mathcal{A} \rightarrow \mathbb{Z}_{+}$nonzero functions on the vectors in $\mathcal{A}$.

Consider the deformations

$$
\begin{aligned}
\mathcal{L}_{\left\{k_{A}\right\}} & =\mathcal{L}_{0}+\sum_{s=2}^{K}(-1)^{s-1}(s-1)\left(\begin{array}{c}
K \\
s
\end{array}\right) \sum_{A \in \mathcal{A}} \frac{\left(k_{A}, s\right)}{\langle A, x\rangle^{s}} \sum_{\alpha} q_{s}(\alpha, A) \partial_{\alpha_{1}} \cdots \partial_{\alpha_{K-s}}, \\
q_{s}(\alpha, A) & =\sum_{\alpha_{K-s+1}, \ldots, \alpha_{K}} a_{\alpha_{1}, \ldots, \alpha_{K}} A_{\alpha_{K-s+1}} \cdots A_{\alpha_{K}}, \quad s=2, \ldots, K,
\end{aligned}
$$

of $\mathcal{L}_{0}$. 
In the context of Theorem 2, the problem on gauge-equivalent deformations of homogeneous differential operators with constant coefficients, posed in [10, takes the following more specific form: describe explicitly all systems $\mathcal{A}$ of nonzero vectors and functions $k_{A}$ on these systems such that the operators $\mathcal{L}_{\left\{k_{A}\right\}}$ are $|k|$-gauge equivalent to the operator $\mathcal{L}_{0}$ via the function $f_{\left\{k_{A}\right\}}(x)$, i.e.,

$$
\operatorname{ad}_{\mathcal{L}_{\left\{k_{A}\right\}}, \mathcal{L}_{0}}^{|k|+1} f_{\left\{k_{A}\right\}}=0, \quad|k|=\sum_{A \in \mathcal{A}} k_{A}, \quad f_{\left\{k_{A}\right\}}(x)=\prod_{A \in \mathcal{A}}\langle A, x\rangle^{k_{A}} .
$$

Partial solutions of this problem are described in the following examples.

Example 1. We may take a system $\mathcal{A}$ consisting of a single nonzero vector, and put $\left\{k_{A}\right\}=k \in \mathbb{Z}_{+}$(this is the result of Theorem 2 and Proposition 2).

Example 2 [3]. Let $x=\left(x_{1}, \ldots, x_{N}\right) \in \mathbb{R}^{N}$, and let

$$
\mathcal{L}_{0} \equiv \Delta=\frac{\partial^{2}}{\partial x_{1}^{2}}+\cdots+\frac{\partial^{2}}{\partial x_{N}^{2}}
$$

be the Laplace operator on $\mathbb{R}^{N}, K=2$. For the role of $\mathcal{A}$ we take the set of positive roots of an arbitrary root system in $\mathbb{R}^{N}$ that corresponds to a finite group $W=\left\langle s_{A}\right\rangle$ generated by reflections

$$
s_{A} x=x-\frac{2\langle A, x\rangle}{\langle A, A\rangle} x
$$

in $\mathbb{R}^{N}$. As $k_{A}$, we take the functions invariant with respect to the action of this group of reflections: $k_{w A}=k_{A}, w \in W$. Since

$$
a_{\alpha_{1} \alpha_{2}}=\left\{\begin{array}{ll}
0, & \alpha_{1} \neq \alpha_{2}, \\
1, & \alpha_{1}=\alpha_{2},
\end{array} \quad q_{2}(\alpha, A)=\langle A, A\rangle,\right.
$$

we obtain the following deformations of the Laplace operator:

$$
\mathcal{L}_{\left\{k_{A}\right\}}=\Delta-\sum_{A \in \mathcal{A}} \frac{k_{A}\left(k_{A}+1\right)}{\langle A, x\rangle^{2}}\langle A, A\rangle .
$$

These deformations coincide with the well-known deformations of the Laplace operator by the Calogero-Moser potentials, and they satisfy the condition of $|k|$-gauge equivalence via the function $f_{\left\{k_{A}\right\}}$; see [3, 7, 8].

As a result, we see that the deformation of the wave operator by the Calogero-Moser potentials $[3,7,8$, and its powers

$$
\left(\square+u_{\left\{k_{A}\right\}}(x)\right)^{s} \equiv\left(\frac{\partial^{2}}{\partial t^{2}}-\Delta+\sum_{A \in \mathcal{A}} \frac{k_{A}\left(k_{A}+1\right)}{\langle A, x\rangle^{2}}\langle A, A\rangle\right)^{s}
$$

are iso-Huygens, and the Huygens principle is fulfilled for these operators if the odd number $N$ satisfies the inequality

$$
2 \sum_{A \in \mathcal{A}} k_{A} \leq N-1-2 s
$$

Remark. As has already been mentioned, the Huygens index $H_{\square}$ of the wave operator on $\mathbb{R}^{1+N}$ is

$$
H_{\square}=\frac{N-1}{2}
$$

if $N$ is odd. Moreover, the Huygens index $H_{\square+u_{\left\{k_{A}\right\}}(x)}$ of the deformation $\square+u_{\left\{k_{A}\right\}}(x)$ of the wave operator reduces, naturally, to

$$
H_{\square+u_{\left\{k_{A}\right\}}(x)}=\frac{N-1}{2}-\sum_{A \in \mathcal{A}} k_{A} .
$$




\section{REFERENCES}

[1] J. Hadamard, Le problème de Cauchy et les équations aux dérivées partielles linéaires hyperboliques, Hermann, Paris, 1932.

[2] N. Kh. Ibragimov, Transformation groups in mathematical physics, "Nauka", Moscow, 1983. (Russian) MR0734307 (85j:58003)

[3] Yu. Yu. Berest and A. P. Veselov, The Huygens principle and integrability, Uspekhi Mat. Nauk 49 (1994), no. 6, 7-78; English transl., Russian Math. Surveys 49 (1994), no. 6, 5-77. MR.1316866 (96a:35003)

[4] K. L. Stellmacher, Ein Beispiel einer Huyghensschen Differentialgleichung, Nachr. Akad. Wiss. Göttingen. Math. Phys. Kl. Math.-Phys. Chem. Abt. 1953, 133-138. MR0060695 (15:710c)

[5] J. E. Lagnese and K. L. Stellmacher, A method of generating classes of Huygens' operators, J. Math. Mech. 17 (1967), no. 5, 461-472. MR0217409 (36:499)

[6] G. Darboux, Sur la représentation sphérique des surfaces, Compt. Rend. (Paris) 94 (1882), 13431345 .

[7] Y. Berest and Y. Molchanov, Fundamental solutions for partial differential equations with reflection group invariance, J. Math. Phys. 36 (1995), 4324-4339. MR.1341994 (96c:35005)

[8] Y. Berest, Hierarchies of Huygens' operators and Hadamard's conjecture, Acta Appl. Math. 53 (1998), no. 2, 125-185. MR.1646583 (99j:58204)

[9] S. P. Khèkalo, Iso-Huygens deformations of the Cayley operator by the general Lagnese-Stellmacher potential, Izv. Ross. Akad. Nauk Ser. Mat. 67 (2003), no. 4, 189-212; English transl., Izv. Math. 67 (2003), no. 4, 815-836. MR2001768 (2004f:35109)

[10] Y. Berest, The problem of lacunas and analysis on root systems, Trans. Amer. Math. Soc. 352 (2000), no. 8, 3743-3776. MR1694280(2001d:58030)

[11] A. M. Gabrièlov and V. P. Palamodov, The Huygens' principle and its generalizations, I. G. Petrovski1. Selected Works. Part I. Systems of Partial Differential Equations. Algebraic Geometry, "Nauka", Moscow, 1986, pp. 449-456; English transl., Classics of Soviet Mathematics, vol. 5, Gordon and Breach Publ., Amsterdam, 1996. MR0871873 (88f:01059) MR.1677652 (99m:01106a)

[12] S. G. Gindikin, The Cauchy problem for strongly homogeneous differential operators, Trudy Moskov. Mat. Obshch. 16 (1967), 181-208; English transl. in Trans. Moscow Math. Soc. 1967 (1968). MR 0227593 (37:3177)

[13] V. M. Babich, Hadamard's ansatz, its analogues, generalizations and applications, Algebra i Analiz 3 (1991), no. 5, 1-37; English transl., St. Petersburg Math. J. 3 (1992), no. 5, 937-972. MR1186234 (93i:35004)

[14] P. Günther, Ein Beispiel einer nichttrivialen Huygensschen Differentialgleichung mit vier unabhängigen Variablen, Arch. Rational Mech. Anal. 18 (1965), 103-106. MR0174865 (30:5056)

[15] M. A. Semenov-Tyan-Shanskiı̆, Harmonic analysis on Riemannian symmetric spaces of negative curvature, and scattering theory, Izv. Akad. Nauk SSSR Ser. Mat. 40 (1976), no. 3, 562-592; English transl., Math. USSR-Izv. 10 (1976), no. 3, 535-563 (1977). MR0467179 (57:7044)

[16] S. Helgason, Integral geometry and multitemporal wave equations, Comm. Pure Appl. Math. 51 (1998), 1035-1071. MR1632583 (99j:58207)

[17] I. M. Gel'fand, S. G. Gindikin, and M. I. Graev, Selected problems in integral geometry, "Dobrosvet", Moscow, 2000. (Russian) MR1795833 (2002d:53100)

[18] E. Ournycheva and B. Rubin, An analogue of the Fuglede formula in integral geometry on matrix spaces, Preprint, Math.FA/0401127, 1, (2004), pp. 1-20. Contemp. Math., vol. 382, Amer. Math. Soc., Providence, RI, 2005, pp. 305-320. MR2175898 (2006g:44005)

[19] I. G. Petrovskiĭ, Selected works. Systems of partial differential equations. Algebraic geometry, "Nauka", Moscow, 1986; English transl., Gordon and Breach Publ., Amsterdam, 1996. MR0871873 (88f:01059) MR:1677652 (99m:01106a)

[20] S. P. Khèkalo, Gauge-equivalent deformations of linear ordinary differential operators with constant coefficients, Zap. Nauchn. Sem. S.-Peterburg. Otdel. Mat. Inst. Steklov. (POMI) 308 (2004), 235251; English transl., J. Math. Sci. (N. Y.) 132 (2006), no. 1, 136-145. MR2092189 (2005g:34226)

[21] The Cayley-Laplace differential operator on the space of rectangular matrices, Izv. Ross. Akad. Nauk Ser. Mat. 69 (2005), no. 1, 195-224; English transl., Izv. Math. 69 (2005), no. 1, 191-219. MR 2128187 (2005m:35009)

[22] M. Kh. Ibragimov and A. O. Oganesyan, Hierarchy of Huygens equations in spaces with a nontrivial conformal group, Uspekhi Mat. Nauk 46 (1991), no. 3, 111-146; English transl., Russian Math. Surveys 46 (1991), no. 3, 137-176. MR1134091 (92m:58137)

[23] S. P. Khèkalo, Stepwise gauge equivalence of differential operators, Mat. Zametki 77 (2005), no. 6, 917-929; English transl., Math. Notes 77 (2005), no. 5-6, 843-854. MR2246966 (2007d:35004) 
[24] L. Gårding, The solution of Cauchy's problem for two totally hyperbolic linear differential equations by means of Riesz integrals, Ann. of Math. (2) 48 (1947), 785-826. MR0022648 (9:240a)

[25] S. P. Khèkalo, Homogeneous differential operators and Riesz potentials in the space of rectangular matrices, Dokl. Akad. Nauk 404 (2005), no. 5, 604-607; English transl. in Russian Acad. Sci. Dokl. Math. MR2256819(2007h:22005)

Kolomna State Pedagogical University, Russia

E-mail address: fmf@kolomna.ru

Received 21/SEP/2007

Translated by THE AUTHOR 